leagues have previously described interneurones in the buccal and abdominal ganglia which mediate excitatory actions at synapses with certain of the follower cells, and inhibitory actions at others. According to Dale's principle, a single neurone releases the same transmitter at all of its terminals, and this seems to hold true in the present case, because both excitatory and inhibitory synaptic potentials resulting from activation of the interneurones can be mimicked in the appropriate follower cells by the application of acetylcholine (ACh).

The differences in response must therefore be determined at the postsynaptic receptor sites, and have been shown by the authors to be associated with different changes in the ionic conductances of the postsynaptic membrane, similar to those observed for excitatory and inhibitory responses in many other systems. Thus, excitation results from a depolarizing postsynaptic potential (EPSP), with a reversal potential of around $-14 \mathrm{mV}$ for cells with a mean resting potential of $-60 \mathrm{mV}$, which probably depends on an increase in membrane conductance to sodium ions because it is reduced or abolished in the presence of low-sodium media. Inhibition, however, is associated with a hyperpolarizing potential (IPSP), which reverses at about -74 $\mathrm{mV}$, and is found to be blocked in the absence of external chloride ions, suggesting that an increase in chloride conductance is involved.

Gardner and Kandel identified two types of excitatory follower cell in the buccal ganglion of Aplysia; one showed a conventional single component EPSP, but in the other type the potential response proved on closer inspection to be diphasic, with a small late inhibitory component following the initial depolarization. This second component could be made more evident by reduction of the membrane potential, which caused it to increase in size. The reversal potential for the first component had a mean of $-10 \mathrm{mV}$, and that for the second component was $-63 \mathrm{mV}$. Both actions appeared to result from direct connexions, because they were unaffected by high calcium solutions which would be expected to reduce the response if it were dependent on conduction in intercalated interneurones.

Iontophoretic applications of $\mathrm{ACh}$ to those follower cells which showed a two-component synaptic potential also caused a diphasic depolarizing-hyperpolarizing response. Moreover, each of the two components could be selectively blocked by different cholinergic blocking agents; hexamethonium, which blocks the EPSP in purely excitatory follower cells but not the IPSP in purely inhibitory follower cells, also blocked the excitatory component in those cells showing diphasic responses without affecting the inhibitory component. Conversely, curare selectively blocked the inhibitory component in dual follower cells. This suggests that the diphasic response in dual follower cells results from the simultaneous combination of ACh with the two types of postsynaptic cholinergic receptor which are at present singly in purely excitatory or inhibitory cells.

A similar "dual" synapse involving both excitatory and inhibitory cholinergic receptors has been previously described by Kandel and his colleagues in the abdominal ganglion of Aplysia, but in this case simultaneous activation of both types of receptor during presynaptic stimulation rarely occurs because of differences in their threshold and rate of densitization. Thus, at low firing rates, pure EPSPs are obtained, and at higher rates pure IPSPs result, presumably because the excitatory receptors are desensitized. Thus the physiological effectiveness of the synapse is determined by the state of sensitization of the two types of receptor.

The two components of the dual synapse in the buccal ganglion also vary in their response to repeated presynaptic stimulation. Although both components are reduced the decrease is greater for the hyperpolarizing component, presumably again because of preferential desensitization at high firing rates, but in this case of the inhibitory receptors. Thus the sign of the buccal ganglion dual synapse, like that of its counterpart in the abdominal ganglion, is frequency dependent, although in the reverse way. Furthermore, it is dependent on the membrane potential, tending to be primarily excitatory near the membrane potential but becoming progressively more inhibitory as the membrane is depolarized by other inputs.

As is pointed out by Gardner and Kandel, the moral of Aplysia's tale is that a wide variety of synaptic actions can be triggered by a single chemical transmitter and the response obtained is dependent on the types of postsynaptic receptor, the combination of receptors and their sequence of activation.

\section{ENVIRONMENTAL HEALTH \\ Moderation is the Word}

from a Correspondent

If the tone of the recent sixth annual conference on trace substances in environmental health at the University of Missouri, June 13-15, is an indicator, the near hysteria that has gripped segments of the scientific community and public at large in the United States during the past several years seems to be gradually easing.

In discussing the geography of insecticides and storage of organochlorine compounds, Dr M. Wasserman (Hebrew University, Jerusalem) pointed out the tendency of biological systems to stabilize internally in spite of qualitative

\title{
Aggregation Factors and Receptors
}

IN Nature New Biology next Wednesday (July 5), Pessac and Defendi describe a set of experiments which strongly support the notion that cells aggregate because they have on their surfaces receptors which bind so-called aggregation factors. Moreover, these workers also suggest that the potential of malignant cells to form solid tumours may be directly related to an increase in the number of surface receptors which bind aggregation factors.

The observation that the medium in which cells have been cultivated promotes cell aggregation-presumably because cells release into their medium aggregation factors and thereby condition it-has been made repeatedly and in many cases aggregation factors or more precisely conditional media have been shown to act on heterologous as well as homologous cells. But how do these factors act? Pessac and Defendi, who favoured the hypothesis that they bind to receptor sites on the cell surface and cross-link one cell to the next, set about answering this question by investigating the properties of four lines of murine lymphoid cells which do not aggregate spontaneously in media lacking serum.

Because medium conditioned by these lymphoid cells does not promote the aggregation of any cells they conclude that the cells of these four lines do not secrete aggregation factors. Furthermore, cells of three of the four lines do not aggregate when placed in medium known to contain aggregation factors. Cells of the fourth line do, however, aggregate under these conditions. Pessac and Defendi conclude therefore that when cells fail to aggregate they either fail to make aggregation factor or lack surface receptors or both, and that aggregation factors and receptors are distinct structurally.

The fact that receptors can be destroyed in situ at the cell surface by proteases whereas aggregation factors are not susceptible to proteolytic enzymes but are inactivated by hyaluronidase also indicates that receptors and factors are chemically distinct. Finally, Pessac and Defendi comment that it may be significant that highly malignant cells aggregate more readily than do weakly malignant cells. 